\title{
ON THE DERIVATIVES OF NEWTONIAN AND LOGARITHMIC POTENTIALS NEAR THE ACTING MASSES*
}

\author{
BY \\ MILDRED M. SULLIVAN
}

1. Introduction. The existing theorems $\dagger$ on the continuity of the derivatives of the potentials of various spreads of acting matter, at points of the spreads, exact more of the densities and surfaces involved than is necessary for the conclusions drawn. A single exception is the work of Petrini, $\ddagger$ who considers necessary and sufficient conditions. The generality of his results, and the incident delicacy of the considerations establishing them, have prevented their becoming widely current. Moreover, they are concerned only with the derivatives of the first two orders in the case of potentials of volume distributions, and of the first order in the case of surface spreads.

Recently, $\S$ Professor Kellogg, in a study of the continuity of harmonic functions defined by their boundary values, and of their derivatives, has shown the usefulness of a simple condition due to Dini.\| The present paper consists in a systematic application of the Dini condition to harmonic functions defined by the densities of the spreads whose potentials they are. Because of the immediate availability of the same methods of proof, theorems have been added on the effect of Hölder conditions on the densities and their derivatives in assuring the existence of Hölder conditions on the potentials and their derivatives, results which are already at hand only for the derivatives of the first order, in the work of Schauder (loc. cit.). The treatment is

* Presented to the Society, October 31, 1931; received by the editors April 20, 1932.

The author desires to make to Professor O. D. Kellogg grateful acknowledgment for the suggestion which led to this paper, and for his helpful interest in its development.

$\dagger$ See, for the earlier literature, L. Lichtenstein, Neuere Entwickelung der Potentialtheorie. Konforme Abbildung, Encyklopädie der mathematischen Wissenschaften, II C 3, pp. 199-209. See also J. Schauder, Potentialtheoretische Untersuchungen, Erste Abhandlung, Mathematische Zeitschrift, vol. 33 (1931), pp. 602-640; L. Lichtenstein, Über einige Hilfssätze der Potentialtheorie, IV, Sächsische Berichte, vol. 82 (1930), pp. 265-344.

$\ddagger$ Les dérivées premières et secondes du potentiel, Acta Mathematica, vol. 31 (1908), pp. 127-332.

$\S$ On the derivatives of harmonic functions on the boundary, these Transactions, vol. 33 (1931), pp. 486-510.

\|Sur la méthode des approximations successives pour les équations aux dérivées partielles $d u$ deuxieme ordre, Acta Mathematica, vol. 25 (1902), p. 224. The form of the condition used in the present paper is slightly more general than that employed by Professor Kellogg. 
given for space of three dimensions, but it is shown that the results are valid also for logarithmic potentials.*

2. Definitions and lemmas. We introduce the following

Definition. Let $f(q)$ be defined on the regular surface element $S \dagger$, and be continuous at the point $p$ of $S$. Let $q$ vary on $S$ in any fixed plane through the normal to $S$ at $p$. Then $f(q)$ is said to satisfy a uniform Dini condition at $p$ if the integral

$$
\int_{0}^{a} \frac{|f(q)-f(p)|}{r} d r, r=\overline{p q}, \ddagger
$$

converges uniformly as to the normal plane chosen. $f(q)$ is said to satisfy a uniform Dini condition on $S$ if it is continuous on $S$ and the convergence of the integral (1) is uniform both as to the point $p$ and as to the direction at $p$.

It will be convenient to use the following class notations. If $S$ is a regular surface element admitting for some orientation of the axes a representation $\zeta=\phi(\xi, \eta)$ where $\phi(\xi, \eta)$ has derivatives of order $n$ which satisfy a uniform Dini condition on $S$ we shall say that $S$ is a regular surface element of class $C^{n+\delta}$. If $f(\xi, \eta)$ has derivatives of order $n$ on $S$ which satisfy a uniform Dini condition there, we shall say that $f(\xi, \eta)$ is of class $C^{n+\delta}$ on $S$. To say that $S$ (or $f(\xi, \eta)$ ) is of class $C^{n+\lambda}$ will mean that the derivatives of $\phi(\xi, \eta)($ or $f(\xi, \eta)$ ) of order $n$ satisfy a uniform Hölder condition with exponent $\lambda$ on $S$.

A region of type $V$ will be understood to mean a closed region of space (consisting of an open continuum and its boundary), partially bounded by the surface $S$ under consideration, but containing no boundary points of $S$, and such that the point $P$ can approach $S$ from only one side while remaining in $V$.

We shall frequently have to consider the product or quotient of two functions each of which satisfies a uniform Dini condition on $S$. We shall need the following lemmas.

LEMMA 1. Let $f$ and $\phi$ be continuous functions satisfying a uniform Dini condition on $S$. Then $f \phi$ satisfies a uniform Dini condition on $S$, and, provided $\phi$ has a positive lower bound, so also does $f / \phi$.

This follows at once from the inequalities

* In a paper entitled Potential functions on the boundary of their regions of definition, these Transactions, vol. 9 (1908), pp. 39-50, Professor Kellogg used a condition of the Dini type in a study of double distributions in the plane. The present results are more general in that less is required of the boundary curve.

$\dagger$ For definition, see Kellogg, Foundations of Potential Theory, Berlin, 1929, p. 105.

$\ddagger$ It is readily shown on the basis of the regularity of $S$ that, for sufficiently small $a$, to each value of $r, 0 \leqq r \leqq a$, there corresponds one and only one point $q$. 


$$
\begin{aligned}
\int_{e}^{a} \frac{|f(q) \phi(q)-f(p) \phi(p)|}{r} d r \\
\quad \leqq \int_{e}^{a} \frac{|\phi(q)||f(q)-f(p)|}{r} d r+\int_{e}^{a} \frac{|f(p)||\phi(q)-\phi(p)|}{r} d r \\
\quad \leqq \max |\phi| \int_{e}^{a} \frac{|f(q)-f(p)|}{r} d r+\max |f| \int_{e}^{a} \frac{|\phi(q)-\phi(p)|}{r} d r
\end{aligned}
$$

together with similar ones for the quotient.

In the same way, we establish

LemMa 2. If $S$ is of class $C^{1+\delta}$, sec $\gamma=\left(1+\phi_{\xi}{ }^{2}+\phi_{\eta}{ }^{2}\right)^{1 / 2}$ satisfies a uniform Dini condition on $S$.

Here $\gamma$ denotes the angle between the normal to $S$ at $q$ and the $\zeta$-axis. As a corollary to these lemmas, we note that if $S$ is in $C^{1+\delta}$ and if $f(q)$ satisfies a uniform Dini condition on $S$, the same is true of $f(q) \sec \gamma$.

We shall also have need of

Lемма 3. Let $S$ be a regular surface element, and let $r^{\prime}$ denote the projection of $r=\overline{p q}$ on the tangent plane to $S$ at $p$. Then if either of the integrals

$$
\int_{0}^{a} \frac{|f(q)-f(p)| d r^{\prime}}{r^{\prime}}, \quad \int_{0}^{a} \frac{|f(q)-f(p)|}{r} d r
$$

converges uniformly as to any parameters, so does the other.

This follows from the facts, first that $r^{\prime} \leqq r$, and secondly that $r \leqq$ arc $\widetilde{p} q<r^{\prime} \max |\sec \gamma|$, the arc being in the plane through $p$ and $q$ containing the normal to $S$ at $p$. The existence of the maximum of sec $\gamma$ is implied in the definition of regular surface element. Thus the above integrals may be used interchangeably in Dini conditions.

It is important to notice the following.

LEMMA 4. The definition of the classes $C^{n+\delta}$ and $C^{n+\lambda}$ is independent of the coördinate system. That is, if $S$ is in either class for one system of orthogonal axes, it is in the same class for any other system of orthogonal axes in which the angle between the tangent plane to the surface and the $\zeta \cdot$ axis has a positive lower. bound.

If $x, y, z$ are the coördinates referred to the given axes and $\xi, \eta, \zeta$ the coordinates referred to any other axes with the specified properties, we have on $S$

$$
\begin{gathered}
\xi=l_{1} x+m_{1} y+n_{1} f(x, y), \quad \eta=l_{2} x+m_{2} y+n_{2} f(x, y), \\
\zeta=l_{3} x+m_{3} y+n_{3} f(x, y),
\end{gathered}
$$


where $z=f(x, y)$ is the given representation of $S$. These equations define a representation $\zeta=\phi(\xi, \eta)$ of $S$ with respect to the second system of axes. We find, then,

$$
\frac{\partial \zeta}{\partial \xi}=-\frac{l_{1} f_{x}+m_{1} f_{y}-n_{1}}{l_{3} f_{x}+m_{3} f_{y}-n_{3}} .
$$

The denominator is the cosine of the angle between the normal to $S$ and the $\zeta$-axis multiplied by $\left(1+f_{x}{ }^{2}+f_{y}{ }^{2}\right)^{1 / 2}$. It has, therefore, a positive lower bound. Then, if $S$ is of class $C^{1+8}$ in the given system of coördinates, it follows that this derivative satisfies a uniform Dini condition on $S$. The same considerations apply to the other derivative of the first order of $\zeta$. Therefore, $S$ is of class $C^{1+8}$ in the second system of coördinates. Similarly, if $S$ is of class $C^{1+\lambda}$ in the given system of coördinates, the derivatives of the first order of $\zeta$ satisfy a uniform Hölder condition on $S$.

In the same way, Lemma 4 can be extended to derivatives of any order. Thus the concept of the class of a regular surface element $S$ is independent of the coördinate system provided no normal to the surface is at right angles to the $\zeta$-axis.

If $S$ is a regular surface element there exists a positive constant $c$ such that if $\sigma$ is the portion of $S$ in a sphere of radius $c$ about any interior point of $S$, then $\sigma$ has a standard representation with tangent-normal axes at any point $p$ of $\sigma .^{*}$ Applying Lemma 4, we see that if $S$ is of class $C^{n+\delta}, \sigma$ is of class $C^{n+\delta}$ with respect to a tangent-normal system of axes at any point $p$ of $\sigma$.

In our theorems on the derivatives of the first order of the potential of a simple distribution we need the following.

LEMMA 5. If $S$ is a regular surface element of class $C^{1+\delta}$ having a standard representation with respect to a tangent-normal system of axes at any interior point p given by $\zeta=\phi(\xi, \eta)$, the integral

$$
\iint_{\sigma^{\prime}} \frac{|\zeta|}{r^{\prime 3}} d S^{\prime}
$$

where $\sigma^{\prime}$ is a circle about $p$ in the $(\xi, \eta)$-plane and $r^{\prime}$ is the projection of $r$ on this plane, converges at $p$, and the convergence is uniform as to $p$.

We cut out from $\sigma^{\prime}$ a circle $c$ of radius $\epsilon$. Then

$$
\iint_{\sigma^{\prime}-c} \frac{|\zeta|}{r^{\prime 3}} d S^{\prime}=\int_{0}^{2 \pi} \int_{0}^{a} \frac{|\zeta|}{r^{\prime 2}} d r^{\prime} d \theta
$$

* See Kellogg, Foundations of Potential Theory, loc. cit., p. 157. 
since the integrand is continuous in $\sigma^{\prime}-c$. This equality holds for any $\epsilon>0$. If the inner integral on the right converges uniformly as to $\theta$ when $\epsilon \rightarrow 0$, the iterated integral approaches a limit, which limit is the value of the improper double integral over $\sigma^{\prime}$, as well as the value of the iterated integral in which $r^{\prime}$ goes from 0 to $a$.

We shall study, then,

$$
\int_{0}^{a} \frac{|\zeta|}{r^{\prime 2}} d r^{\prime}
$$

If we write $\xi=t \cos \theta, \eta=t \sin \theta$,

$$
\begin{aligned}
\zeta & =\zeta(0,0)+\int_{0}^{r^{\prime}} \frac{d \zeta}{d t} d t \\
& =0+\int_{0}^{r^{\prime}}\left[\phi_{\xi}(t \cos \theta, t \sin \theta) \cos \theta+\phi_{\eta}(t \cos \theta, t \sin \theta) \sin \theta\right] d t .
\end{aligned}
$$

Hence,

$$
\begin{aligned}
|\zeta| & \leqq \int_{0}^{r^{\prime}}\left\{\left|\phi_{\xi}(t)\right|+\left|\phi_{\eta}(t)\right|\right\} d t \\
& =\int_{0}^{r^{\prime}} t \frac{d}{d t}(I+J) d t,
\end{aligned}
$$

where

$I(t, \theta)=\int_{0}^{t} \frac{\left|\phi_{\xi}\left(r^{\prime} \cos \theta, r^{\prime} \sin \theta\right)\right|}{r^{\prime}} d r^{\prime}, J(t, \theta)=\int_{0}^{t} \frac{\left|\phi_{\eta}\left(r^{\prime} \cos \theta, r^{\prime} \sin \theta\right)\right|}{r^{\prime}} d r^{\prime}$.

By hypothesis, $I$ and $J$ vanish with $t$ uniformly as to $\theta$. Therefore,

$$
\int_{a}^{a} \frac{|\zeta|}{r^{\prime 2}} d r^{\prime} \leqq \int_{0}^{a} \frac{1}{r^{\prime 2}} \int_{0}^{r^{\prime}} t \frac{d}{d t}(I+J) d t d r^{\prime}
$$

Integrating by parts, we have for the integral on the right

$$
\begin{aligned}
& -\left.\frac{1}{r^{\prime}} \int_{0}^{r^{\prime}} t \frac{d}{d t}(I+J) d t\right|_{\cdot} ^{a}+\int_{0}^{a} \frac{1}{r^{\prime}} r^{\prime} \frac{d}{d r^{\prime}}(I+J) d r^{\prime} \\
& =\frac{1}{\epsilon} \int_{0}^{a} t \frac{d}{d t}(I+J) d t-\frac{1}{a} \int_{0}^{a} t \frac{d}{d t}(I+J) d t+(I+J)_{r^{\prime}-a}-(I+J)_{r^{\prime}-\mathbf{c}}
\end{aligned}
$$

The last term vanishes with $\epsilon$. This is true of the first term, also; since, by the law of the mean, 


$$
\begin{aligned}
\frac{1}{\epsilon} \int_{0}^{\epsilon} t \frac{d}{d t}(I+J) d t & =\frac{\bar{t}}{\epsilon} \int_{0}^{\epsilon} \frac{d}{d t}(I+J) d t \\
& =\frac{\bar{t}}{\epsilon}(I+J)_{t-\epsilon}
\end{aligned}
$$

where $0<\bar{t} / \epsilon<1$. Therefore,

$$
\begin{aligned}
\int_{0}^{a} \frac{|\zeta|}{r^{\prime 2}} d r^{\prime} & \leqq(I+J)_{r^{\prime}=a}-\frac{1}{a} \int_{0}^{a} t \frac{d}{d t}(I+J) d t \\
& \leqq(I+J)_{r^{\prime}=a}
\end{aligned}
$$

since the derivatives of $I$ and $J$ with respect to $t$ are never negative. It follows therefore, not only that

$$
\iint_{\sigma^{\prime}} \frac{|\zeta|}{r^{\prime 8}} d r^{\prime}
$$

converges everywhere on $S$, but that this integral approaches 0 with the radius of $\sigma^{\prime}$, uniformly.

3. Existence and continuity of the derivatives of the first order of the potential of a simple distribution.

Tangential derivatives. We prove the following theorem:

TheORem I. Let $S$ be a regular surface element of class $C^{1+\delta}$. Let $\sigma$, the density of a simple distribution on $S$, satisfy a uniform Dini condition at the interior point $p$ of $S$. Then the derivative of the potential $U$ at $P$ in the direction of any tangent at $p$ approaches a limit as $P$ approaches $p$ along the normal. If $\sigma$ satisfies a uniform Dini condition over a closed portion of $S$ containing no boundary points of $S$, the limits of such derivatives are approached uniformly as to $p$ on such a portion.

We shall restrict ourselves to the portion of $S$ contained in a sphere of radius $c$ about $p$, such that we have a single representation of the whole piece with a tangent-normal system of axes at any point of the piece. We have seen that such a positive constant $c$ exists uniformly as to $p$. As the potential of the rest of $S$ is analytic in a neighborhood of $p$, we may neglect it and assume that all of $S$ is contained in this sphere.

We take a tangent-normal system of axes at $p$, choosing the $x$-axis in the direction in which we are taking the derivative and the $y$-axis in the perpendicular tangential direction. Let $P$ be a point on the $z$-axis. Then for $z \neq 0$,

$$
\frac{\partial U}{\partial x}=\iint_{S} \sigma \frac{\xi}{r^{3}} d S=\iint_{S^{\prime}} \sigma \sec \gamma \frac{\xi}{r^{3}} d S^{\prime},
$$


$S^{\prime}$ being the projection of $S$ on the $(x, y)$-plane and $\gamma$ the angle between the normal to $S$ and the $z$-axis. This derivative may be written

$$
\frac{\partial U}{\partial x}=I+J
$$

where

$$
\begin{gathered}
I=\iint_{\sigma^{\prime}} \sigma \sec \gamma \frac{\xi}{r^{3}} d S^{\prime}, \quad J=\iint_{S^{\prime} \sigma^{\prime}} \sigma \sec \gamma \frac{\xi}{r^{3}} d S^{\prime}, \\
r^{2}=\xi^{2}+\eta^{2}+(z-\zeta)^{2},
\end{gathered}
$$

$\sigma^{\prime}$ being a small circle of radius $a$ about $p$ in the $(x, y)$-plane. Then, for any fixed $\sigma^{\prime}, J$ is continuous, and corresponding to any $\epsilon>0$, there is a $\delta$ depending on $\epsilon$ and $\sigma^{\prime}$ only, such that

$$
\left|J\left(z_{2}\right)-J\left(z_{1}\right)\right|<\epsilon / 3 \text { when } 0<z_{1}<\delta, 0^{-}<z_{2}<\delta .
$$

We write

where

$$
I=I_{1}+I_{2}
$$

$$
I_{1}=\sigma(p) \iint_{\sigma^{\prime}} \frac{\xi}{r^{3}} d S^{\prime}, I_{2}=\iint_{\sigma^{\prime}}[\sigma(q) \sec \gamma-\sigma(p)] \frac{\xi}{\gamma^{3}} d S^{\prime},
$$

and compare $I_{1}$ with the integral

$$
\sigma(p) \iint_{\sigma^{\prime}} \frac{\xi}{\rho^{3}} d S^{\prime} \text { where } \rho^{2}=\xi^{2}+\eta^{2}+z^{2} .
$$

This integral vanishes since the integrand has equal and opposite values at $(\xi, \eta)$ and $(-\xi, \eta)$. Hence,

$$
\begin{aligned}
I_{1} & =\sigma(p) \iint_{\sigma^{\prime}} \xi\left(\frac{1}{r^{3}}-\frac{1}{\rho^{3}}\right) d S^{\prime} \\
& =\sigma(p) \iint_{\sigma^{\prime}} \frac{\xi \zeta(2 z-\zeta)}{r \rho(r+\rho)}\left(\frac{1}{r^{2}}+\frac{1}{r \rho}+\frac{1}{\rho^{2}}\right) d S^{\prime} .
\end{aligned}
$$

Since

$$
|\xi| \leqq r^{\prime}=\left(\xi^{2}+\eta^{2}\right)^{1 / 2},|z| \leqq \rho,|z-\zeta| \leqq r,|2 z-\zeta| \leqq r+\rho, r^{\prime} \leqq \rho, r^{\prime} \leqq r,
$$

it follows that

$$
\left|I_{1}\right| \leqq 3 \max |\sigma| \iint_{\sigma^{\prime}} \frac{|\zeta|}{r^{\prime 3}} d S^{\prime}
$$


By Lemma 5, the last integral converges. It follows that we can so choose $\sigma^{\prime}$ that

$$
\iint_{\sigma^{\prime}} \frac{|\zeta|}{r^{\prime 3}} d S^{\prime}<\frac{1}{18 \max |\sigma|} \epsilon
$$

unless $\max |\sigma|=0$ (in which case $I_{1} \equiv 0$ ). Therefore, by choosing $a$ small enough we can make $\left|I_{1}\right|<\epsilon / 6$, independently of $z$. Then

$$
\left|I_{1}\left(z_{2}\right)-I_{1}\left(z_{1}\right)\right|<\epsilon / 3 \text {. }
$$

As to the second integral, we have

$$
\begin{aligned}
\left|I_{2}\right| & \leqq \iint_{\sigma^{\prime}}|\sigma(q) \sec \gamma-\sigma(p)| \frac{1}{r^{\prime 2}} d S^{\prime} \\
& \leqq \int_{0}^{2 \pi} \int_{0}^{a} \frac{|\sigma(q) \sec \gamma-\sigma(p)|}{r^{\prime}} d r^{\prime} d \theta .
\end{aligned}
$$

By Lemmas 1 and 2, the inner integral converges at $p$, uniformly as to $\theta$. Therefore, by taking $a$ small enough, $a$ depending on $p$ in this case, we can make

$$
\int_{0}^{a} \frac{|\sigma(q) \sec \gamma-\sigma(p)|}{r^{\prime}} d r^{\prime}<\epsilon /(12 \pi)
$$

independently of $\theta$. Then we shall have

$$
\left|I_{2}\left(z_{2}\right)-I_{2}\left(z_{1}\right)\right|<\epsilon / 3 \text {. }
$$

Having chosen $a$ we can, as we have seen, by taking $0<z_{1}<\delta, 0<z_{2}<\delta$, make

$$
\left|J\left(z_{2}\right)-J\left(z_{1}\right)\right|<\epsilon / 3 \text {. }
$$

Therefore, for $z_{1}$ and $z_{2}$ so restricted,

$$
\left|\frac{\partial U\left(z_{2}\right)}{\partial x}-\frac{\partial U\left(z_{1}\right)}{\partial x}\right|<\epsilon,
$$

and the derivative approaches a limit as $P$ approaches $p$ along the normal at $p$.

The only inequality which depends on the point $p$ is that for $I_{2}$. If $\sigma$ satisfies a uniform Dini condition on a closed portion of $S$ this inequality can be made independent of the position of $p$ on this portion of $S$. Therefore, under these conditions, the approach of the derivative to its limit along the normal will be uniform on this part of $S$, both as to the point $p$, and as to the tangential direction of differentiation. 
Normal derivatives. In studying the normal derivative at $P$ as $P$ approaches $p$ along the normal, we need assume only that $\sigma$ is bounded and integrable on $S$ and continuous at $p$. We assume that $S$, as in the case of tangential derivatives, is a regular surface element of class $C^{1+\delta}$.

When $z \neq 0$ the normal derivative at $P(0,0, z)$ is given by

$$
\frac{\partial U}{\partial z}=\iint_{S} \sigma \frac{\zeta-z}{r^{3}} d S=\iint_{S^{\prime}} \sigma \sec \gamma \frac{\zeta-z}{r^{3}} d S^{\prime}
$$

where $S^{\prime}$ is the projection of $S$ on the $(x, y)$-plane. Let $U^{\prime}$ be the potential of a plane lamina occupying the area $S^{\prime}$ and having the density $\sigma \sec \gamma$. Then

$$
\frac{\partial U^{\prime}}{\partial z}=-\iint_{S^{\prime}} \sigma \sec \gamma \frac{z}{\rho^{3}} d S^{\prime} \text { where } \rho^{2}=\xi^{2}+\eta^{2}+z^{2} .
$$

This derivative, as is well known, approaches $\mp 2 \pi \sigma(p)$ according as $P$ approaches $p$ along the positive or the negative $z$-axis.

We consider the difference

$$
\begin{aligned}
\frac{\partial U}{\partial z}-\frac{\partial U^{\prime}}{\partial z} & =\iint_{S^{\prime}} \sigma \sec \gamma\left[\frac{\zeta-z}{r^{3}}+\frac{z}{\rho^{3}}\right] d S^{\prime} \\
& =\iint_{S^{\prime}} \sigma \sec \gamma\left[\frac{\zeta}{r^{3}}-z\left(\frac{1}{r^{3}}-\frac{1}{\rho^{3}}\right)\right] d S^{\prime} \\
& =I_{1}+I_{2}
\end{aligned}
$$

where

$$
\begin{aligned}
& I_{1}=\iint_{\sigma^{\prime}} \sigma \sec \gamma\left[\frac{\zeta}{r^{3}}-z\left(\frac{1}{r^{3}}-\frac{1}{\rho^{3}}\right)\right] d S^{\prime}, \\
& I_{2}=\iint_{S^{\prime}-\sigma^{\prime}} \sigma \sec \gamma\left[\frac{\zeta}{r^{3}}-z\left(\frac{1}{r^{3}}-\frac{1}{\rho^{3}}\right)\right] d S^{\prime} .
\end{aligned}
$$

The integral $I_{2}$ is continuous in $z$ for fixed $\sigma^{\prime}$. As to $I_{1}$, if we use the algebraic identity already employed in connection with the tangential derivatives, and the inequalities

$$
|z| \leqq \rho,|2 z-\zeta| \leqq r+\rho, r^{\prime} \leqq r, r^{\prime} \leqq \rho,
$$

we find that

$$
\left|I_{1}\right| \leqq 4 \max |\sigma \sec \gamma| \iint_{\sigma^{\prime}} \frac{|\zeta|}{r^{\prime 3}} d S^{\prime} .
$$

Thus, by Lemma $5, I_{1}$ can be made arbitrarily small by sufficiently restricting $\sigma^{\prime}$. 
We conclude that the difference (4) is continuous at $z=0$. The value of this difference at $p$ is given by the convergent integrals

$$
\iint_{S^{\prime}} \sigma \sec \gamma \frac{\zeta}{r^{3}} d S^{\prime}=\iint_{S} \sigma \frac{\zeta}{r^{3}} d S=\iint_{S} \sigma\left(\frac{\partial}{\partial z} \frac{1}{r}\right)_{p} d S .
$$

Using the values of the limits of the derivative (3), we have as limits of the derivative (2) when $P$ approaches $p$ from the positive or negative side

$$
\left(\frac{\partial U}{\partial z}\right)_{+}+2 \pi \sigma(p)=\left(\frac{\partial U}{\partial z}\right)_{-}-2 \pi \sigma(p)=\iint_{S} \sigma\left(\frac{\partial}{\partial z} \frac{1}{r}\right)_{p} d S .
$$

These limits are approached uniformly as to $p$ on any closed interior portion of $S$ on which $\sigma$ is continuous.

Letting $n$ denote the direction of the normal in the positive sense to $S$ at $p$, we may state the results obtained, in

Theorem II. Let $S$ be a regular surface element of class $C^{1+\delta}$. Let $\sigma$, the density of a simple distribution on $S$, be continuous at $p$. Then the normal derivative of the potential of this distribution approaches a limit as $P$ approaches $p$ along the normal to $S$ at $p$ from either side and these limits are

$$
\begin{aligned}
& \frac{\partial U}{\partial n_{+}}=-2 \pi \sigma(p)+\iint_{S} \sigma\left(\frac{\partial}{\partial n} \frac{1}{r}\right)_{p} d S, \\
& \frac{\partial U}{\partial n_{-}}=+2 \pi \sigma(p)+\iint_{S} \sigma\left(\frac{\partial}{\partial n} \frac{1}{r}\right)_{p} d S .
\end{aligned}
$$

These limits are approached uniformly as to $p$ on any closed portion of $S$, containing no boundary points of $S$, on which $\sigma$ is continuous.

Derivatives in any direction. It follows from Theorems I and II, that if $S$ is a regular surface element of class $C^{1+\delta}$ and $\sigma$ satisfies a uniform Dini condition at $p$, the derivative in any direction approaches a limit as $P$ approaches $p$ along the normal at $p$. If $\sigma$ satisfies a uniform Dini condition on a closed interior portion of $S$, the derivative of $U$ in a fixed direction approaches its limits uniformly along the normals on this portion of $S$. We shall now prove

ThEOREM III. Let $S$ be a regular surface element of class $C^{1+\delta}$. Let $\sigma$ satisfy a uniform Dini condition on $S$. Then the potential $U$ of the distribution of density $\sigma$ on $S$ has derivatives of the first order which, when defined on $S$ by their limits, are continuous in any region of type $V$.

The difficulty of the situation arises from the fact that, in the absence of hypotheses assuring curvatures of $S$, we cannot count on the existence of a field of normals to $S$. The following lemma, however, will be all that is needed: 
Let $Q$ be a point on the normal to $S$ at $p$, and let $\sigma$ denote a sphere about $Q$. Then there exists a sphere $\sigma^{\prime}$ about $p$, such that every point $P$ of $\sigma^{\prime}$ lies on a normal to $S$ which meets $\sigma$.

Let $\alpha$ denote the radius of a sphere $\sigma^{\prime}$ about $p$. We shall show that $\alpha$ can be chosen small enough to meet the requirements of the lemma. As a first restriction, $2 \alpha$ is to be less than the distance from the interior point $p$ to the boundary of $S$. Let $P$ be a point of $\sigma^{\prime}$. The largest sphere about $P$ which contains in its interior no points of $S$, has on its surface at least one interior point of $S$. Let $q$ be such a point. Then $P q$, being a minimal segment from $P$ to $S$, is normal to $S$ at $q$. Moreover, $\overline{P q} \leqq \overline{P p} \leqq \alpha$, and hence $\overline{p q} \leqq 2 \alpha$. Furthermore, because of the continuity of the direction cosines of the normal to $S$, the normals to $S$ at points in $\sigma^{\prime}$ make with the normal at $p$, angles having a maximum $\eta$, which approaches 0 with $\alpha$. It follows that the greatest distance from $Q$ to the normal $P q$ does not exceed $2 \alpha+\overline{p Q}$ sin $\eta$. As $\eta \rightarrow 0$ with $\alpha, \alpha$ can be chosen positive and so small that the normal $P q$ will certainly meet $\sigma$.

Turning now to the proof of the theorem, we consider the continuity of any derivative of $U$ at an interior point $p$ of $S$. Let $F(P)$ denote the derivative in question. $F(P)$ approaches its limits along normals, uniformly. There thus corresponds to any $\epsilon>0$, a one-sided neighborhood $N$ of $p$, in which $F(P)$ differs from its limiting values on normals by less than $\epsilon / 4$. Let $Q$ be a point of the normal at $p$ in $N$. Then since $F(P)$ is continuous at $Q$, there is a sphere $\sigma$ about $Q$ and in $N$, such that if $P^{\prime}$ is in $\sigma$,

$$
\left|F\left(P^{\prime}\right)-F(Q)\right|<\epsilon / 4 .
$$

If $P$ is any point in the sphere $\sigma^{\prime}$, which corresponds, by the lemma, to $\sigma$, we may take $P^{\prime}$ as a point of the normal $P q$. We then have the following inequalities, resulting from the uniform approach along the normals:

$$
\begin{gathered}
\left|F\left(P^{\prime}\right)-F(q)\right|<\epsilon / 4, \\
|F(P)-F(q)|<\epsilon / 4, \\
|F(Q)-F(p)|<\epsilon / 4 .
\end{gathered}
$$

It follows from these that if $P$ is any point in $\sigma^{\prime}$,

$$
|F(P)-F(p)|<\epsilon,
$$

and the derivative is thus continuous at $p$. The rest of the theorem follows from the analytic character of the potential at points not on $S$.

4. Existence and continuity of the potential of a double distribution at points of the distribution. We shall consider the potential of a double distribution on a regular surface element $S$ of class $C^{1+\delta}$. The moment $\mu$ is to be bounded and integrable. The potential is given by the integral 


$$
U=\iint_{S} \mu \frac{\partial}{\partial \nu} \frac{1}{r} d S
$$

For the sake of completeness, we show first that this improper integral converges on $S$ under the present assumptions. Using as the field of integration the projection of $S$ on the tangent plane at $p$, this amounts to verifying that

$$
\lim \iint_{S^{\prime}-\sigma^{\prime}} \mu \frac{\partial}{\partial \nu} \frac{1}{r} \sec \gamma d S^{\prime}=\lim \iint_{S^{\prime}-\sigma^{\prime}} \frac{\zeta-\xi \phi_{\xi}-\eta \phi_{\eta}}{r^{3}} d S^{\prime}
$$

exists, $\sigma^{\prime}$ being a circle about $p$, whose radius tends to 0 . But since $\mu$ is bounded and integrable,

$$
\lim \iint_{S^{\prime}-\sigma^{\prime}} \mu \frac{\zeta}{r^{3}} d S^{\prime}
$$

exists, by Lemma 5. Moreover,

$$
\iint_{S^{\prime}-\sigma^{\prime}}\left|\mu \frac{-\xi \phi_{\xi}-\eta \phi_{\eta}}{r^{3}}\right| d S^{\prime} \leqq \max |\mu| \int_{0}^{2 \pi} \int_{\epsilon}^{a} \frac{\left|\phi_{\xi}\right|+\left|\phi_{\eta}\right|}{r^{\prime}} d r^{\prime} d \theta
$$

where $\epsilon$ is the radius of $\sigma^{\prime}$, and $a$ the maximum radius of $S^{\prime}$. As the integral on the right converges, because $S$ is in $C^{1+\delta}$, the rest of the integral under discussion converges absolutely. Thus the integral for $U$ has a meaning at points of $S$. We denote its value by $U_{0}$.

If we introduce $\cos \alpha, \cos \beta, \cos \gamma$, the direction $\operatorname{cosines}$ of the normal to $S$ at $q(\xi, \eta, \zeta), U$ takes the form

$U=-\iint_{S} \mu \cos \alpha \frac{\partial}{\partial x} \frac{1}{r} d S-\iint_{S} \mu \cos \beta \frac{\partial}{\partial y} \frac{1}{r} d S-\iint_{S} \mu \cos \gamma \frac{\partial}{\partial z} \frac{1}{r} d S$.

We suppose $S$ is referred to tangent-normal axes at $p$. The first two terms on the right then represent tangential derivatives of simple distributions on $S$, while the third is the normal derivative of such a distribution. If $\mu$ is continuous at $p, \mu \cos \alpha$ and $\mu \cos \beta$ satisfy a uniform Dini condition there, as is readily verified, and $\mu \cos \gamma$ is continuous. From these facts and from Theorems I and II we obtain

THEOREM IV. Let $S$ be a regular surface element of class $C^{1+\delta}$. Let $\mu$, the moment of a double distribution on $S$, be bounded and integrable on $S$ and continuous: at the interior point $p$ of $S$. Then, as $P$ approaches $p$ along the normal to $S$ at $p$ from either side, the potential $U$ of the double distribution approaches limits given by 


$$
\begin{aligned}
& U_{+}=2 \pi \mu(p)+U_{0}, \\
& U_{-}=-2 \pi \mu(p)+U_{0} .
\end{aligned}
$$

On any closed portion of $S$ containing no boundary points of $S$, on which $\mu$ is continuous, these limits are approached uniformly.

By the reasoning used in proving Theorem III we have

THEOREM V. Let $S$ be a regular surface element of class $C^{1+\delta}$. Let $\mu$, the moment of a double distribution on $S$, be continuous on $S$. Then the potential $U$ of the distribution, when defined on $S$ by its limits, is continuous in any region of type $V$.

5. Korn's identities. In studying the derivatives of order $n$ of the potentials of simple and double distributions we shall use two identities due to Korn.* We shall state these identities in the following lemmas.

LEMMA 6. If $S$ is a regular surface element of class $C^{\prime \prime}$ and $\sigma$ is continuously differentiable on $S$, the identity

$$
\begin{aligned}
\frac{\partial}{\partial x} \iint_{S} \sigma \frac{1}{r} d S= & \int_{\Gamma} \sigma \frac{\cos (\nu, \eta) \cos (s, \zeta)-\cos (\nu, \zeta) \cos (s, \eta)}{r} d s \\
& +\iint_{S} \frac{1}{r}\left\{\sigma_{\xi}-l\left[\sigma\left(l_{\xi}+m_{\eta} \dot{+} n_{\zeta}\right)+\sigma_{\xi} l+\sigma_{\eta} m+\sigma_{\xi} n\right]\right\} d S \\
& +\iint_{S} \sigma l \frac{\partial}{\partial \nu} \frac{1}{r} d S
\end{aligned}
$$

(where $\Gamma$ is the boundary of $S$, and $l, m, n$ are the direction cosines of the normal at $q$ ) holds for points not on $S$.

LemMa 7. If $S$ is a regular surface element and $\mu$ is continuously differentiable on $S$, the identity

$$
\begin{aligned}
\frac{\partial}{\partial x} \iint_{S} \mu \frac{\partial}{\partial \nu} \frac{1}{r} d S= & \int_{\Gamma} \mu \frac{\cos (s, \zeta) \cos (r, \eta)-\cos (s, \eta) \cos (r, \zeta)}{r^{2}} d s \\
& +\frac{\partial}{\partial x} \iint_{S} l_{\mu_{\xi}} \frac{1}{r} d S+\frac{\partial}{\partial y} \iint_{S} l_{\mu_{\eta}} \frac{1}{r} d S+\frac{\partial}{\partial z} \iint_{S} l_{\mu_{\xi}} \frac{1}{r} d S \\
& +\iint_{S} \mu_{\xi} \frac{\partial}{\partial \nu} \frac{1}{r} d S
\end{aligned}
$$

(where $\Gamma$ is the boundary of $S$, and $l, m, n$ are the direction cosines of the normal at $q$ ) holds for points not on $S$.

* A. Korn, Potentialtheorie, vol. I, Berlin, 1899, pp. 36-38, pp. 40-42. 
6. Existence and continuity of the derivatives of order $n$ of the potentials of simple and double distributions. We have already obtained, in Theorem III, sufficient conditions that the derivatives of the first order of a simple distribution on $S$ be continuous when defined on $S$ by their limiting values. Using Theorems III and V and Lemma 7, we find that if $S$ is a regular surface element of class $C^{1+\delta}$ and $\mu$ is of class $C^{1+\delta}$ on $S$, the potential of the double distribution of moment $\mu$ on $S$ is continuously differentiable in a region of type $V$.

We shall now prove the general theorems for derivatives of order $n$.

Theorem VI. Let $S$ be a regular surface element of class $C^{n+\delta}$. Let $\sigma$, the density of a simple distribution on $S$, be of class $C^{n-1+\delta}$. Then $U$, the potential of this distribution, has continuous derivatives of order $n$ in any region of type $V$ when they are defined on $S$ by their limits.

TheOREM VII. Let $S$ be a regular surface element of class $C^{n+\delta}$. Let $\mu$, the moment of a double distribution on $S$, be of class $C^{n+\delta}$. Then $U$, the potential of this distribution, has continuous derivatives of order $n$ in any region of type $V$ when they are defined on $S$ by their limits.

We prove these theorems, already established for $n=1$, by induction. We assume that both theorems are true when $n$ is replaced by $n-1$.

Let $S$ have the standard representation $\zeta=\phi(\xi, \eta)$ with respect to the $(\xi, \eta, \zeta)$-axes. Then $\phi$ is of class $C^{n+\delta}$. For the potential of Theorem VI, by Lemma 6, we have

$$
\begin{aligned}
\frac{\partial U}{\partial x}= & \int_{\Gamma} \sigma \frac{\cos (\nu, \eta) \cos (s, \zeta)-\cos (\nu, \zeta) \cos (s, \eta)}{r} d s \\
& +\iint_{S}\left\{\sigma_{\xi}-l\left[\sigma\left(l_{\xi}+m_{\eta}\right)+\sigma_{\xi} l+\sigma_{\eta} m\right]\right\} \frac{1}{r} d S \\
& +\iint_{S} l \sigma \frac{\partial}{\partial \nu} \frac{1}{r} d S,
\end{aligned}
$$

with similar expressions for $\partial U / \partial y$ and $\partial U / \partial z$.

The first term in (5) is analytic at all points not on $\Gamma$. Therefore, this term has continuous derivatives of all orders in the closed region $V$. The second term is the potential of a simple distribution on $S$ with density of class $C^{n-2+\delta}$. By our assumption that the theorem is true when $n$ is replaced by $n-1$, this term has continuous derivatives of order $n-1$ in $V$ when they are defined on $S$ by their limits. The same is true of the third term, since it is the potential of a double distribution on $S$ with moment of class $C^{n-1+\delta}$. Therefore, the whole expression (5) has continuous derivatives of order $n-1$ in $V$ 
when they are defined on $S$ by their limits. The expressions for the other two derivatives of $U$ can be discussed in a similar manner, with the result that they also have continuous derivatives of order $n-1$ in $V$. Therefore, $U$ has continuous derivatives of order $n$ in $V$ when they are defined on $S$ by their limiting values.

Turning to the proof of Theorem VII, we study the potential

$$
U=\iint_{S} \mu \frac{\partial}{\partial \nu} \frac{1}{r} d S
$$

for which, by Lemma 7,

$$
\begin{aligned}
\frac{\partial U}{\partial x}= & \int_{\Gamma} \mu \frac{\cos (s, \zeta) \cos (r, \eta)-\cos (s, \eta) \cos (r, \zeta)}{r^{2}} d s \\
& +\frac{\partial}{\partial x} \iint_{S} l \mu_{\xi} \frac{1}{r} d S+\frac{\partial}{\partial y} \iint_{S} l \mu_{\eta} \frac{1}{r} d S+\iint_{S} \mu_{\xi} \frac{\partial}{\partial \nu} \frac{1}{r} d S,
\end{aligned}
$$

with similar expressions for $\partial U / \partial y$ and $\partial U / \partial z$.

The reasoning employed for Theorem VI now yields the desired result.

We have proved then, that if the theorems are true for derivatives of order $n-1$, they are true for derivatives of order $n$. We know that they hold for $n=1$. Therefore, they hold for any positive integral $n$.

7. On the scope of the sufficient conditions established above. We have just found sufficient conditions that surface potentials have continuous derivatives of order $n$. It is natural to ask if we can place lighter conditions upon the spreads without impairing the conclusions of Theorems VI and VII. If we can produce examples satisfying slightly lighter hypotheses and show that the derivatives of order $n$ do not exist or are not continuous, it follows that these lighter hypotheses cannot furnish sufficient conditions for the existence and continuity of derivatives of order $n$.

Let us consider the derivatives of order $n$ of a simple distribution. Is it possible to get a set of conditions for the existence and continuity of these derivatives in which we require of $\sigma$ only that it be of class $C^{n-1}$ ?

We let $S$ be a circular lamina of radius $a<1$. The plane of $S$ will be the $(\xi, \eta)$-plane. We write $\xi=r^{\prime} \cos \theta, \eta=r^{\prime} \sin \theta$. We consider the densities

$$
\sigma=-\frac{r^{\prime n-1} \cos \theta}{\log r^{\prime}}, \text { if } n \text { is odd, } \sigma=-\frac{r^{\prime n-1}}{\log r^{\prime}}, \text { if } n \text { is even, }
$$

where $r^{\prime}$ is measured from the center of $S$. We see that $\sigma$ has continuous derivatives of order $n-1$ on $S$ and that these derivatives do not satisfy a Dini condition at $r^{\prime}=0$. We consider. 


$$
\left.\frac{\partial^{n} U}{\partial x^{n}}\right|_{P}
$$

where $P$ is the point $(0,0, z), z \neq 0$, that is, a point on the normal to $S$ at the center $p$. We shall prove that this derivative becomes infinite as $P$ approaches $p$.

In fact,

$$
\left.\frac{\partial^{n} U}{\partial x^{n}}\right|_{P}=\iint_{S} \sigma \frac{\partial^{n}}{\partial x^{n}} \frac{1}{r} d S,
$$

and

$$
\left.\frac{\partial^{n}}{\partial x^{n}} \frac{1}{r}\right|_{x=y=0}=(-1)^{n} \frac{C_{n} P_{n}(u)}{r^{n+1}}, u=-\frac{\xi}{r}, r^{2}=r^{\prime 2}+z^{2},
$$

where $C_{n}>0$, and where $P_{n}(u)$ is the Legendre polynomial of order $n$. Then

$$
\begin{aligned}
\left.\frac{\partial^{n} U}{\partial x^{n}}\right|_{P} & =(-1)^{n} C_{n} \iint_{S} \sigma \frac{P_{n}(u)}{r^{n+1}} d S \\
& =C_{n} \iint_{S} \sigma \frac{P_{n}\left(\frac{\xi}{r}\right)}{r^{n+1}} d S .
\end{aligned}
$$

If we break $S$ into the circle of radius $\alpha z$ and the remaining annular region, where $\alpha$ is a suitably chosen constant and $\alpha z<a$, it can be shown that, when the density $\sigma$ is the function given above, the integral over the first of these regions approaches 0 with $z$ while the integral over the second region becomes infinite. Hence, the derivative in question becomes infinite as $P$ approaches $p$.

Therefore, $\sigma$ being of class $C^{n-1}$ does not insure the existence of the derivatives of order $n$ no matter how smooth the surface $S$.

By similar reasoning it can be shown that $S$ being of class $C^{n}$ does not insure the existence of the derivatives of order $n$ of a simple distribution on $S$ no matter how smooth the density. In proving this we consider the distribution of unit density on the surface given by

$$
\zeta=\frac{r^{\prime n}}{\log r^{\prime}}, \text { if } n \text { is odd, } \zeta=\frac{r^{\prime n} \cos \theta}{\log r^{\prime}}, \text { if } n \text { is even, }
$$

where

$$
r^{\prime 2}=\xi^{2}+\eta^{2}, \quad \xi=r^{\prime} \cos \theta, \quad \eta=r^{\prime} \sin \theta .
$$

Turning now to double distributions, it can be shown, by considering the distribution on a circular lamina with moment $\mu$ defined by 


$$
\mu=-\frac{r^{\prime n}}{\log r^{\prime}}, \text { if } n \text { is odd, } \mu=-\frac{r^{\prime n} \cos \theta}{\log r^{\prime}} \text {, if } n \text { is even, }
$$

that $\mu$ being of class $C^{n}$ does not insure the existence of the derivatives of order $n$ of the potential of a double distribution on $S$ no matter how smooth $S$.

Using a distribution of moment $\xi$ on the surface given by

$$
\zeta=\frac{r^{\prime n}}{\log r^{\prime}}, \text { if } n \text { is odd, } \zeta=\frac{r^{\prime n} \cos \theta}{\log r^{\prime}}, \text { if } n \text { is even, }
$$

it can be shown that $S$ being of class $C^{n}$ does not insure the existence of the derivatives of order $n$ of the potential of a double distribution on $S$, no matter how smooth the moment.

It thus appears unlikely that materially lighter conditions exist which are at once simple and sufficient for the existence and continuity of the derivatives.

8. Hölder conditions on the derivatives of surface distributions. It has been proved* that if $S$ is of class $C^{1+\lambda}$ and $\sigma$ is of class $C^{\lambda^{\prime}}$, the derivatives of the first order of the simple distribution of density $\sigma$ on $S$ satisfy a uniform Hölder condition, in a closed region of type $V$, with exponent $\kappa$, where $0<\kappa$ $\leqq \lambda, \kappa \leqq \lambda^{\prime}$. If $S$ is of class $C^{1+\lambda}$ and $\mu$, the moment of a double distribution on $S$, is of class $C^{1+\lambda^{\prime}}$, the derivatives of the first order of the potential of this distribution satisfy a uniform Hölder condition in a region of type $V$ with exponent $\kappa$. We shall now establish general theorems for Hölder conditions on the derivatives of order $n$.

THEOREM VIII. Let $S$ be a regular surface element of class $C^{n+\lambda}$. Let $\sigma$, the density of a simple distribution on $S$, be of class $C^{n-1+\lambda}$. Then the derivatives of order $n$ of the potential of this distribution when defined on $S$ by their limiting values satisfy a uniform Hölder condition in any region of type $V$ with exponent $\kappa$, where $0<\kappa \leqq \lambda, \kappa \leqq \lambda^{\prime}$.

ThEOREM IX. Let $S$ be a regular surface element of class $C^{n+\lambda}$. Let $\mu$, the moment of a double distribution on $S$, be of class $C^{n+\lambda}$. Then the derivatives of order $n$ of the potential of this distribution when defined on $S$ by their limiting values satisfy a uniform Hölder condition in any region of type $V$ with exponent $\kappa$, where $0<\kappa \leqq \lambda, \kappa \leqq \lambda^{\prime}$.

* See Schauder, loc. cit. Although in the case of the derivatives of the first order, the present methods do not yield more general results than those of Schauder, in the case of the potential of a double distribution itself, they yield a lighter condition on the surface than that employed by him. See Theorem X, p. 156. 
As in an earlier instance, we use the method of induction, assuming that both theorems are true when $n$ is replaced by $n-1$. Then, turning first to the proof of Theorem VIII for $n=n$, we consider the identity (5) for $\partial U / \partial x$.

The first term is analytic at all points not on $\Gamma$, and so in $V$. The second term is the potential of a simple distribution on $S$ with density of class $C^{n-2+x}$. Therefore, this potential has continuous derivatives of order $n-1$ which satisfy a uniform Hölder condition in $V$ with exponent $\kappa$. The third term is the potential of a double distribution on $S$ with moment of class $C^{n-1+x}$. Therefore, the derivatives of order $n-1$ of this potential satisfy a uniform Hölder condition in $V$ with exponent $\kappa$. Hence, the derivative of $U$ with respect to $x$ has continuous derivatives of order $n-1$ which satisfy a uniform Hölder condition in $V$ with exponent $\kappa$. The same is true of the other two derivatives of $U$. Therefore, the derivatives of $U$ of order $n$ satisfy a uniform Hölder condition in $V$ with exponent $k$.

Similar reasoning applied to (6) yields the desired result in the case of Theorem IX.

Since both theorems have been established for $n=1$, it follows that they hold for any positive integral $n$.

9. Hölder conditions on the potentials of surface distributions. We have been concerned with the existence of Hölder conditions on the derivatives of potentials of simple and double distributions on regular surfaces. We now take up the question of such conditions on the potentials themselves.

For a simple distribution, it is known that a distribution with bounded integrable density $\sigma$, on a regular surface element $S$, has a potential which satisfies a uniform Hölder condition with any given exponent less than 1 . In fact, the present methods yield the result for such a potential

$$
\left|U_{2}-U_{1}\right| \leqq 2 \pi \max |\sigma \sec \gamma| r_{12} \log \frac{R e^{6}}{r_{12}}, r_{12} \leqq R,
$$

where $R$ is the inaximum chord of $S$.

We therefore turn at once to the potential of a double distribution, establishing first

LEMMA 8. Let $S$ be a regular surface element of class $C^{1+\delta}$. Then the integral

$$
\iint_{S}\left|\frac{\partial}{\partial \nu} \frac{1}{r}\right| d S
$$

is bounded in any region of type $V$.*

\footnotetext{
* Schauder (loc. cit.) has proved a similar lemma, less general than this, in that $S$ is required to be of class $C^{1+\lambda}$.
} 
As we have seen in connection with the proof of Theorem III, there is a neighborhood $N$ of any interior closed portion of $S$ with the property that through any point of $N$ there passes a normal to $S$ at an interior point of $S$. This neighborhood can be so chosen as to include all points of $S$ in $V$. The points of $V$ not in $N$ are distant at least $\delta$ from $S, \delta$ being a positive constant. At such points the integral (7) is bounded. In fact

$$
\frac{1}{\delta^{2}} \iint_{S} d S
$$

is such a bound.

We may assume then that the parameter point $P$ in (7) is on the normal to $S$ at the interior point $p$. We take a tangent-normal system of axes at $p$ and denote by $\sigma$ a portion of $S$ having a standard representation with these axes. This representation exists uniformly as to $p$. Then

$$
\iint_{S-\sigma}\left|\frac{\partial}{\partial \nu} \frac{1}{r}\right| d S
$$

is bounded uniformly as to $p$.

For the rest of the integral, we have

$$
\begin{aligned}
\iint_{\sigma}\left|\frac{\partial}{\partial \nu} \frac{1}{r}\right| d S & =\iint_{\sigma^{\prime}} \frac{\left|z-\zeta+\phi_{\xi \xi}+\phi_{\eta} \eta\right|}{r^{3}} d S^{\prime} \\
& \leqq I_{1}+I_{2}+I_{3},
\end{aligned}
$$

where

$$
\begin{gathered}
I_{1}=\iint_{\sigma^{\prime}} \frac{|z|}{\rho^{3}} d S^{\prime}, I_{2}=\iint_{\sigma^{\prime}}|z|\left|\frac{1}{r^{3}}-\frac{1}{\rho^{3}}\right| d S^{\prime}, \\
I_{3}=\iint_{\sigma^{\prime}} \frac{\left|-\zeta+\phi_{\xi} \xi+\phi_{\eta} \eta\right|}{r^{\prime 3}} d S^{\prime}, \\
r^{\prime 2}=\xi^{2}+\eta^{2}, \rho^{2}=r^{\prime 2}+z^{2},
\end{gathered}
$$

$\sigma^{\prime}$ being the projection of $\sigma$ on the tangent plane at $p$. Then, since

$$
\iint_{\sigma^{\prime}} \frac{z}{\rho^{3}} d S^{\prime}=\Omega
$$

where $\Omega$ is the solid angle subtended at $P(0,0, z)$ by the flat element of surface $\sigma^{\prime}$,

$$
I_{1} \leqq 2 \pi \text {. }
$$

Also, 


$$
I_{2} \leqq 3 \iint_{\sigma^{\prime}} \frac{|\zeta|}{r^{\prime 3}} d S^{\prime}
$$

By Lemma 5, this integral is bounded and the bound can be taken independent of $p$. The integral $I_{3}$ is bounded, as we have noted at the beginning of $\$ 4$.

Therefore,

$$
\iint_{\sigma}\left|\frac{\partial}{\partial \nu} \frac{1}{r}\right| d S
$$

is bounded uniformly as to $p$. It follows that (7) is bounded in $V$.

We now prove

THEOREM X. Let $S$ be a regular surface element of class $C^{1+\delta}$. Let $\mu$, the moment of a double distribution on $S$, be of class $C^{\lambda}$. Then the potential of this distribution, when defined on $S$ by its limits, satisfies a uniform Hölder condition with exponent $\lambda$ in any region of type $V$.

As in Lemma 8, we shall restrict ourselves to points of $V$ in the neighborhood $N$, since the potential is analytic in the rest of $V$. We let $P_{1}$ be a point of $V$ on the normal to $S$ at the interior point $p_{1}$. We shall now assume that all of $S$ is given by the portion contained in a sphere of radius $c$ about $p_{1}$ and having a standard representation with tangent-normal axes at $p_{1}$, since the potential of the rest of $S$ will be analytic at $P_{1}$. Such a representation exists uniformly as to $p_{1}$. We let $P_{2}$ be a second point of $V$ so restricted that the parallel through $P_{2}$ to $P_{1} p_{1}$ meets $S$ in the interior point $p_{2}$.

We write

$$
U=U_{1}+U_{2}
$$

where

$$
U_{1}=\mu\left(p_{1}\right) \iint_{S} \frac{\partial}{\partial \nu} \frac{1}{r} d S, U_{2}=\iint_{S}\left[\mu(q)-\mu\left(p_{1}\right)\right] \frac{\partial}{\partial \nu} \frac{1}{r} d S .
$$

Since $U_{1}$ is the potential of a double distribution of constant moment on $S$, it has continuous derivatives of the first order in $V$, by Theorem VII.

We have then only to prove that $U_{2}$ satisfies a uniform Hölder condition with exponent $\lambda$. We denote by $\sigma$ the portion of $S$ in a circular cylinder of radius $2 r_{12}$ whose axis is the normal to $S$ at $p_{1}, r_{12}$ being the distance $\overline{P_{1} P_{2}}$, and write

$$
U_{2}\left(P_{2}\right)-U_{2}\left(P_{1}\right)=\Delta_{1}+\Delta_{2},
$$

where 


$$
\begin{aligned}
& \Delta_{1}=\iint_{\sigma}\left[\mu(q)-\mu\left(p_{1}\right)\right]\left[\frac{\partial}{\partial \nu} \frac{1}{r}\right]_{2} d S-\iint_{\sigma}\left[\mu(q)-\mu\left(p_{1}\right)\right]\left[\frac{\partial}{\partial \nu} \frac{1}{r}\right]_{1} d S, \\
& \Delta_{2}=\iint_{S-\sigma}\left[\mu(q)-\mu\left(p_{1}\right)\right]\left[\frac{\partial}{\partial \nu} \frac{1}{r}\right]_{2} d S \\
& \quad-\iint_{S-\sigma}\left[\mu(q)-\mu\left(p_{1}\right)\right]\left[\frac{\partial}{\partial \nu} \frac{1}{r}\right]_{1} d S,
\end{aligned}
$$

the subscripts indicating that the coördinates of $P_{1}$ and $P_{2}$ are to be substituted for $x, y$, and $z$. Then

$$
\left|\Delta_{1}\right| \leqq \max \left|\mu(q)-\mu\left(p_{1}\right)\right|\left\{\left.\iint_{\sigma}\left|\frac{\partial}{\partial \nu} \frac{1}{r}\right|_{2}\left|d S+\iint_{\sigma}\right| \frac{\partial}{\partial \nu} \frac{1}{r}\right|_{1} \mid d S\right\} .
$$

Since the integral in the above inequality is bounded in $V$, by Lemma 8 , and since $\mu$ satisfies a uniform Hölder condition on $S$, it follows that

$$
\left|\Delta_{1}\right| \leqq K_{1} r_{12}^{\lambda}
$$

$K_{1}$ being an appropriate constant.

The remaining difference, $\Delta_{2}$, may be written in the form

$$
\Delta_{2}=\int_{s_{1}}^{s_{2}} \iint_{S-\sigma}\left[\mu(q)-\mu\left(p_{1}\right)\right] \frac{\partial^{2}}{\partial s \partial \nu} \frac{1}{r} d S d s
$$

where the integration with respect to $s$ is from $P_{1}$ to $P_{2}$ along the segment joining them, and where the integrand is continuous in the field of integration. From this it follows that

$$
\left|\Delta_{2}\right| \leqq 10(1+2 M) A \int_{s_{1}}^{s_{2}} \iint_{S-\sigma} r_{1}^{\lambda} \frac{1}{r^{3}} d S d s,
$$

where $M$ is the maximum of $\left|\phi_{\xi}\right|$ and $\left|\phi_{\eta}\right|$ and $A$ is the coefficient in the Hölder condition on $\mu$. Here $r$ is measured from a point on $P_{1} P_{2}$, and $r_{1}$ from $p_{1}$. If we denote by $r_{1}^{\prime}$ and $r^{\prime}$ the projections on the tangent plane at $p_{1}$ of $r_{1}$ and $r$ respectively, we have the inequalities

$$
r_{1} \leqq \max \sec \gamma r_{1}^{\prime}, r^{\prime} \leqq r, r_{1}^{\prime} \leqq 2 r^{\prime} .
$$

Hence, 


$$
\begin{aligned}
\left|\Delta_{2}\right| & \leqq 10(1+2 M) A(\max \sec \gamma)^{\lambda} \int_{s_{1}}^{s_{2}} \iint_{S-\sigma} r_{1}^{\prime \lambda-3}\left(\frac{r_{1}^{\prime}}{r^{\prime}}\right)^{3} d S d s \\
& \leqq 80(1+2 M) A(\max \sec \gamma)^{\lambda} \int_{s_{1}}^{s_{2}} \iint_{S-\sigma} r_{1}^{\prime \lambda-3} d S d s \\
& \leqq 80(1+2 M) A(\max \sec \gamma)^{\lambda} \int_{s_{1}}^{s_{2}} \int_{0}^{2 \pi} \int_{2 r_{12}}^{a} r_{1}^{\prime \lambda-2} d r^{\prime} d \theta d s \\
& \leqq \frac{160(1+2 M) \pi}{2^{1-\lambda}(1-\lambda)} A(\max \sec \gamma)^{\lambda} r_{12}^{\lambda} \\
& \leqq K_{2}{ }^{\lambda} r_{12} .
\end{aligned}
$$

Combining the inequalities for $\Delta_{1}$ and $\Delta_{2}$, we see that $U_{2}$ satisfies a uniform Hölder condition with exponent $\lambda$ in $V$. It follows that the same is true of $U$.

10. The existence and continuity of derivatives of volume potentials. The derivatives of the first order of the potential due to a volume distribution of bounded and integrable density in a bounded volume are continuous throughout space as is well known. As a preliminary to the study of the derivatives of higher order, we define the Dini condition in a region of space.

Definition. Let $f(Q)$ be defined in the closed region $V$, and continuous at the point $P$ of $V$. Let $Q$ vary along a ray through $P$. Then if the integral

$$
\int_{0}^{a} \frac{|f(Q)-f(P)|}{r} d r, r=\overline{P Q},
$$

converges at $P$ uniformly as to the direction of the ray chosen, $f(Q)$ is said to satisfy a uniform Dini condition at $P$. If $f(Q)$ is continuous in $V$ and if the integral (8) converges at every point of $V$ uniformly both as to $P$ and as to the direction of the integration at $P, f(Q)$ is said to satisfy a uniform Dini condition in $V$.

In case $P$ is a boundary point of $V$, a given ray may contain both points of $V$ and points not in $V$. In this case, the above integral is to be understood as the Lebesgue integral over the closed set of points of the interval $(0, a)$, of the ray in $V$. Since the integrand is non-negative; the convergence of the integral is equivalent to the summability of the integrand, and the uniformity of the convergence means that the integral vanishes with $a$, uniformly as to the direction of the ray. The condition at a boundary point is evidently fulfilled if it is possible so to extend the definition of $f(Q)$ to a neighborhood of the boundary point that it is fulfilled by the extended function.

We shall adopt for functions defined in a region of space the class notations given in $\$ 2$ for a function defined on a surface. 
Our object is the establishment of the following theorem.

THEOREM XI. Let $V$ be a regular closed region of space whose boundary $S$ contains a regular surface element $\Sigma$ of class $C^{n-1+\delta}$, and let $\kappa$, the density of a distribution in $V$, be of class $C^{n-2+\delta}, n \geqq 2$. If $n>2$ let $\kappa$ be of class $C^{n-2+\delta}$ on $\Sigma$ as well as in $V .{ }^{*}$ Let $V^{\prime}$ be a closed region of space partly bounded by $\Sigma$, but containing no boundary points of $\Sigma$ or other points of $S$. Then the potential of this distribution has continuous derivatives of order $n$ in $V^{\prime}$ when they are defined on $\Sigma$ by their limits.

We note that the region $V^{\prime}$, except for boundary points in $\Sigma$, may be entirely interior to $V$, or entirely exterior to $V$. The two cases require different treatment and will therefore be taken up separately. We remark that we may once and for all confine ourselves to the portion of $V$ inside a certain sphere which cuts off from $\Sigma$ a portion having a standard representation, $\zeta=\phi(\xi, \eta)$, where $\phi(\xi, \eta)$ is in class $C^{n-1+\delta}$, when referred to axes tangent and normal to $\Sigma$ at the center of the sphere. For later purposes we note that a single radius will serve for this sphere for all points on any closed portion of $\Sigma$ containing no boundary points of $\Sigma$. The justification of confining ourselves to such a portion of $V$ lies first in the fact that the distribution on the rest of $V$ is analytic at the interior points of the sphere, and secondly that exactly the same methods as those here used may be applied to points of $V^{\prime}$ farther from $\Sigma$.

We shall consider then a region $V$ bounded by a spherical surface and a portion of $\Sigma$. We prove the theorem first for $n=2$ and then extend it by induction. We may confine ourselves to one derivative of the second order, since the others may be treated by exactly the same methods.

Considering first the case where $V^{\prime}$ is in $V$, we take a point $P_{1}$ in $V^{\prime}$, and denote by $\kappa_{1}$ the density at $P_{1}$. We break up the integral into two parts,

where

$$
U=\iiint_{V}{ } \frac{1}{r} d V=U_{1}+U_{2},
$$

* A function defined in a volume may satisfy a uniform Dini condition at a boundary point of the volume, and yet fail to satisfy a Dini condition, as defined for functions of position on a surface, at this point of the bounding surface. For example, the values on the surface of the function

$$
\begin{aligned}
f(Q) & =\frac{1}{\log \log \frac{1}{\zeta} \log \frac{1}{\zeta}}, \quad \zeta \geqq 0, \\
& =0, \quad \zeta \leqq 0,
\end{aligned}
$$

considered in the volume bounded by $\zeta=r^{\prime 2}, r^{\prime}=a, a<1$, and $\zeta=-1$, where $r^{\prime 2}=\xi^{2}+\eta^{2}$, fail to satisfy a Dini condition at the origin, although the integral taken along a ray converges uniformly there. 


$$
U_{1}=\kappa_{1} \iiint_{V} \frac{1}{r} d V, U_{2}=\iiint_{V}\left(\kappa-\kappa_{1}\right) \frac{1}{r} d V .
$$

The derivative of the first may be written, with the help of Green's Theorem,

$$
\frac{\partial^{2} U_{1}}{\partial x^{2}}=-\kappa_{1} \frac{\partial}{\partial x} \iiint_{V} \frac{\partial}{\partial \xi} \frac{1}{r} d V=\kappa_{1} \frac{\partial}{\partial x} \iint_{S} \cos (\nu, x) \frac{1}{r} d S .
$$

The differentiation of $U_{2}$ at the point $P_{1}$ may in the present case be carried out under the integral sign because of the sufficiently rapid vanishing of $\left(\kappa-\kappa_{1}\right) .^{*}$ We may therefore write

$$
\text { (9) }\left.\frac{\partial^{2} U}{\partial x^{2}}\right|_{1}=\kappa_{1}\left[\frac{\partial}{\partial x} \iint_{S} \cos (\nu, x) \frac{1}{r} d S\right]_{1}+\iiint_{V}\left(\kappa-\kappa_{1}\right)\left[\frac{\partial^{2}}{\partial x^{2}} \frac{1}{r}\right]_{1} d V \text {, }
$$

the subscript indicating that the coördinates of the corresponding point are to be substituted for $x, y$, and $z$.

Let $P_{2}$ be a second point near $P_{1}$. We write

$$
\left.\frac{\partial^{2} U}{\partial x^{2}}\right|_{2}-\left.\frac{\partial^{2} U}{\partial x^{2}}\right|_{1}=\Delta_{1}+\Delta_{2}
$$

where

$$
\begin{aligned}
& \Delta_{1}=\kappa_{2}\left[\frac{\partial}{\partial x} \iint_{S} \cos (\nu, x) \frac{1}{r} d S\right]_{2}-\kappa_{1}\left[\frac{\partial}{\partial x} \iint_{S} \cos (\nu, x) \frac{1}{r} d S\right]_{1}, \\
& \Delta_{2}=\iiint_{V}\left(\kappa-\kappa_{2}\right)\left[\frac{\partial^{2}}{\partial x^{2}} \frac{1}{r}\right]_{2} d V-\iiint_{V}\left(\kappa-\kappa_{1}\right)\left[\frac{\partial^{2}}{\partial x^{2}} \frac{1}{r}\right]_{1} d V .
\end{aligned}
$$

The integral in $\Delta_{1}$ is the potential of a simple spread on $S$ whose density $\cos (\nu, x)$ is in $C^{\delta}$ on $\Sigma$, and hence, by Theorem III, it has continuous derivatives in $V^{\prime}$. Since $\kappa$ is also continuous, there corresponds to any $\epsilon>0$ a $\delta^{\prime}>0$ depending on $\epsilon$ alone, such that for $\overline{P_{1} P_{2}}<\delta^{\prime}$,

$$
\left|\Delta_{1}\right|<\epsilon / 4 \text {. }
$$

In the discussion of $\Delta_{2}$, we introduce a sphere $\sigma$, of radius $a$ about $P_{1}$, and impose a second restriction $\overline{P_{1} P_{2}}<a / 2$, on $P_{2}$. Then, because of the Dini condition on $\kappa$, uniform in $V$, and the fact that

$$
\left|\frac{\partial^{2}}{\partial x^{2}} \frac{1}{r}\right| \leqq \frac{2}{r^{3}}
$$

it is possible to restrict $a$ so that

* Petrini, loc. cit., p. 135. 


$$
\begin{aligned}
& \left|\iiint_{\sigma}\left(\kappa-\kappa_{1}\right)\left[\frac{\partial^{2}}{\partial x^{2}} \frac{1}{r}\right]_{1} d V\right|<\epsilon / 4, \\
& \left|\iiint_{\sigma}\left(\kappa-\kappa_{2}\right)\left[\frac{\partial^{2}}{\partial x^{2}} \frac{1}{r}\right]_{2} d V\right|<\epsilon / 4,
\end{aligned}
$$

independently of the position of $P_{2}$ in the sphere $\overline{P_{1} P_{2}}<a / 2$. Thus if we write

$$
\Delta_{2}=\Delta_{21}+\Delta_{22}
$$

in the first term of which the field of integration is $\sigma$, we have

$$
\left|\Delta_{21}\right|<\epsilon / 2 \text {. }
$$

The second term, $\Delta_{22}$, is the difference in values at $P_{2}$ and $P_{1}$ of an integral whose integrand is continuous in the variables of integration and the coördinates of the parameter point for $\overline{P_{1} P_{2}}<a / 2$. Hence, if $a$ is fixed, there exists a $\delta^{\prime \prime}>0$ such that if $\overline{P_{1} P_{2}}<\delta^{\prime \prime}$,

$$
\left|\Delta_{22}\right|<\epsilon / 4
$$

Thus, if $a$ is a fixed positive number, such that $\left|\Delta_{21}\right|<\epsilon / 2$, which we have seen is possible, independently of $P_{2}$, and if $\delta$ is the least of the positive numbers $\delta^{\prime}, a / 2, \delta^{\prime \prime}$, then for $\overline{P_{1} P_{2}}<\delta$,

$$
\left|\frac{\partial^{2} U}{\partial x^{2}}\right|_{2}-\left.\frac{\partial^{2} U}{\partial x^{2}}\right|_{1}|\leqq| \Delta_{1}|+| \Delta_{21}|+| \Delta_{22} \mid<\epsilon .
$$

Accordingly, the continuity of this derivative in $V^{\prime}$ is established in the case in which $V^{\prime}$ is in $V$.

Suppose now that $V^{\prime}$ is exterior to $V$ except for common boundary points which are interior points of $\Sigma$. We shall show that the derivative in question approaches limits uniformly along normals to $\Sigma$ on any closed portion of $\Sigma$ including no boundary points of $\Sigma$. The reasoning of Theorem III will then establish the continuity of this derivative in $V^{\prime}$.

Let $p$ be an interior point of $\Sigma$, and let $P$ be a point in $V^{\prime}$ on the normal to $\Sigma$ at $p$. If the $(x, y)$-plane is chosen tangent to $\Sigma$ at $p$, with the positive $z$-axis through $P$, the value of the derivative at $P$ is given by

$$
\frac{\partial^{2} U}{\partial x^{2}}=\iiint_{V}\left(\kappa-\kappa_{0}\right) \frac{\partial^{2}}{\partial x^{2}} \frac{1}{r} d V+\kappa_{0} \frac{\partial}{\partial x} \iint_{S} \cos (\nu, x) \frac{1}{r} d S,
$$

where $\kappa_{0}$ is the density at $p$. Here, the second term is continuous in $V^{\prime}$ as we have already seen, and so approaches a limit as $P$ approaches $p$ along the normal, uniformly in $V^{\prime}$. The first term we write as $I_{1}+I_{2}$ where 


$$
I_{1}=\iiint_{\sigma}\left(\kappa-\kappa_{0}\right) \frac{\partial^{2}}{\partial x^{2}} \frac{1}{r} d V, \quad I_{2}=\iiint_{V-\sigma}\left(\kappa-\kappa_{0}\right) \frac{\partial^{2}}{\partial x^{2}} \frac{1}{r} d V
$$

$\sigma$ being the portion of $V$ in a sphere of radius $a$ about $p$. A first restriction on $a$ is that it be less than the distance from $p$ to the boundary of $\Sigma$. For the first integral, we have

$$
\begin{aligned}
\left|I_{1}\right| & =\left|\iiint_{\sigma}\left(\kappa-\kappa_{0}\right)\left[\frac{3(\xi-x)^{2}}{r^{5}}-\frac{1}{r^{3}}\right] d V\right| \leqq 2 \iiint_{\sigma} \frac{\left|\kappa-\kappa_{0}\right|}{r^{3}} d V \\
& =2 \iiint_{\sigma} \frac{\left|\kappa-\kappa_{0}\right|}{r_{0}^{8}} \frac{r_{0}^{3}}{r^{3}} d V,
\end{aligned}
$$

where $r_{0}$ is measured from $p$ to the point of integration, and $r$ is measured from $P$. The ratio $r_{0} / r$ will be greatest when $r$ is the projection of $r_{0}$ on a plane parallel to the $(x, y)$-plane, which may occur for points of $\sigma$ with positive $z$-coördinates. However, $r_{0} / r$ will never exceed $k=\max \sec \gamma$, where $\gamma$ is the angle between two normals to $\Sigma$ at points of $\sigma$. This number $k$ is independent of $p$. We have then

$$
\left|I_{1}\right| \leqq 2 k^{3} \iiint_{0} \frac{\left|\kappa-\kappa_{0}\right|}{r_{0}^{3}} d V
$$

which is convergent, and vanishes uniformly with $a$, because $\kappa$ is in $C^{\delta}$.

Accordingly, $a$ can be chosen so that the oscillation of $I_{1}$ can be made arbitrarily small independently of the position of $P$ on the normal. Then, with $a$ fixed, $I_{2}$ is continuous at $p$. Hence, the derivative approaches a limit along the normal. As the inequalities involved can be made independent of $p$, the approach is uniform in $V^{\prime}$.

Theorem XI is thus established for $n=2$. In extending the proof, we make use of Green's Theorem to write the derivative of the potential

$$
U=\iiint_{V} \kappa \frac{1}{r} d V
$$

in the form

$$
\frac{\partial U}{\partial x}=\iint_{S} \kappa \cos (\nu, x) \frac{1}{r} d S+\iiint_{V} \kappa_{\xi} \frac{1}{r} d V,
$$

valid for points $P(x, y, z)$ not on $S$ for $\kappa$ in $C^{\prime}$. We assume that Theorem XI has been established for derivatives of order $n-1$. The first term in (11) is the potential of a simple distribution on $S$ with density of class $C^{n-2+\delta}$ on $\Sigma$. By Theorem VI, this potential has continuous derivatives of order $n-1$ in 
$V^{\prime}$. By our assumption that the theorem holds when $n-1$ is substituted for $n$, the second term has continuous derivatives of order $n-1$ in $V^{\prime}$. Therefore $\partial U / \partial x$ has continuous derivatives of order $n-1$ in $V^{\prime}$. The same applies to $\partial U / \partial y$ and $\partial U / \partial z$, so that all the derivatives of $U$ with respect to $x, y$, and $z$, of order $n$, exist and are continuous in $V^{\prime}$.

Since the theorem is true for derivatives of the second order it follows that it is true for derivatives of any higher order.

11. Hölder conditions on the derivatives of volume potentials. The potential of a distribution of bounded and integrable density in a bounded volume $V$ has continuous derivatives of the first order which satisfy a uniform Hölder condition with exponent $\lambda$, where $\lambda$ is any number such that

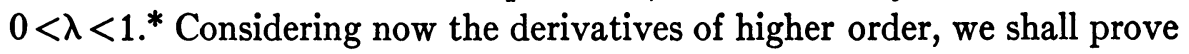

THEOREM XII. Let $V$ be a regular closed region of space whose boundary $S$ contains a regular surface element $\Sigma$ of class $C^{n-1+\lambda}$, and let $\kappa$, the density of a distribution in $V$, be of class $C^{n-2+\lambda^{\prime}}$. Let $V^{\prime}$ be a closed region of space partly bounded by $\Sigma$ but containing no boundary points of $\Sigma$ or other points of $S$. Then the derivatives of order $n$ of the potential of this distribution, when defined on $\Sigma$ by their limiting values, satisfy a uniform Hölder condition in $V^{\prime}$ with exponent $\lambda^{\prime \prime}$, where $0<\lambda^{\prime \prime} \leqq \lambda, \lambda^{\prime \prime} \leqq \lambda^{\prime}$.

The proof is analogous to that of Theorem XI. Our induction begins with the case $n=2$, and to fix ideas, we consider the second derivative with respect to $x$, first for the case in which $V^{\prime}$ is in $V$. By equation (9), this derivative, regarded as a function of $P$, satisfies

$$
\frac{\partial^{2} U}{\partial x^{2}}=\kappa(P) \frac{\partial}{\partial x} \iint_{S} \cos (\nu, x) \frac{1}{r} d S+\iiint_{V}[\kappa(Q)-\kappa(P)] \frac{\partial^{2}}{\partial x^{2}} \frac{1}{r} d V .
$$

The derivative in the first term on the right satisfies a uniform Hölder condition in $V^{\prime}$ with exponent $\lambda$, and by the reasoning of Lemma 1 , the first term then satisfies a uniform Hölder condition in $V^{\prime}$ with exponent $\lambda^{\prime \prime}$.

In establishing the same property for the second term we shall need the following lemma: Let $\sigma$ be the portion of $V$ in a sphere of radius $\alpha$ about an interior point of $V^{\prime}, \alpha$ being so restricted that $\sigma$ contains no points of $S$ other than points of $\Sigma$. Then any derivative of the second order of the potential

$$
\iiint_{0} \frac{1}{r} d V
$$

\footnotetext{
* See Korn, Sur les équations de l'élasticité, Annales de l'Ecole Normale, (3), vol. 24 (1907), p. 28. Although the bounding surfaces of the volumes considered there have bounded curvatures, the reasoning can be extended so as to apply to any bounded volume.
} 
is bounded at the center of the sphere, and this bound depends only on $\Sigma$.

If the sphere is entirely interior to $V$ such a bound is seen at once to exist. We assume then that there are points of $\Sigma$ in the sphere. Through $P$, the center of the sphere, there will pass a normal to $\Sigma$ at one of these points $p$. If we denote by $t$ the larger of the two regions into which the tangent plane at $p$ divides the sphere, we may write

$$
\frac{\partial^{2}}{\partial x^{2}} \iiint_{0} \frac{1}{r} d V=\frac{\partial^{2}}{\partial x^{2}} \iiint_{t} \frac{1}{r} d V+J,
$$

where

$$
|J| \leqq \iiint\left|\frac{\partial^{2}}{\partial x^{2}} \frac{1}{r}\right| d V \leqq 2 \iiint \frac{1}{r^{3}} d V,
$$

the integral being taken over a set of points containing all points in $\sigma$, or $t$, but not in both (i.e. in $\sigma+t-\sigma \cdot t$ ). But if we refer to tangent-normal axes at $p$, and denote by $\rho$ the projection of $r$ on the tangent plane this set is surely included between $\zeta=-A \rho^{1+\lambda}$ and $\zeta=+A \rho^{1+\lambda}$. Therefore,

$$
|J| \leqq 4 \pi \int_{0}^{\alpha} \int_{A-\rho^{1+\lambda}}^{A \rho^{1+\lambda}} \frac{1}{r^{3}} d \zeta \rho d \rho \leqq 8 \pi k^{3} \int_{0}^{\alpha} \int_{0}^{A \rho^{1+\lambda}} \frac{1}{r^{\prime 3}} d \zeta \rho d \rho,
$$

where $k=\max \sec \gamma$ on $\Sigma$, and where $r^{\prime}$ is measured from $p$ to the point of integration. It follows that

$$
|J| \leqq \frac{8 \pi A k^{3}}{\lambda} \alpha^{\lambda} \leqq \frac{8 \pi A k^{3} R^{\lambda}}{\lambda}
$$

where $R$ is the maximum chord of $V$.

Considerations of an elementary nature establish the fact that the integral over $t$ has derivatives of the second order which are bounded at $P$, and that these bounds can be taken independent of $\alpha$ and the position of the dividing plane.

Returning now to the proof of the theorem for $n=2$ and $V^{\prime}$ in $V$, we consider the difference of the values of the second term in the above equality at two interior points, $P_{1}$ and $P_{2}$. If we denote by $\sigma$ the portion of $V$ in a sphere of radius $2 r_{12}$ about $P_{1}$, this difference may be written as $\Delta_{1}+\Delta_{2}$, where

$$
\begin{aligned}
\Delta_{1}=\iiint_{\sigma}\left[\kappa(Q)-\kappa\left(P_{2}\right)\right]\left[\frac{\partial^{2}}{\partial x^{2}} \frac{1}{r}\right]_{2} d V & \\
& -\iiint_{\sigma}\left[\kappa(Q)-\kappa\left(P_{1}\right)\right]\left[\frac{\partial^{2}}{\partial x^{2}} \frac{1}{r}\right]_{1} d V,
\end{aligned}
$$




$$
\begin{aligned}
\Delta_{2}=\iiint_{V-\sigma}\left[\kappa(Q)-\kappa\left(P_{2}\right)\right]\left[\frac{\partial^{2}}{\partial x^{2}} \frac{1}{r}\right]_{2} d V \\
\\
-\iiint_{V-\sigma}\left[\kappa(Q)-\kappa\left(P_{1}\right)\right]\left[\frac{\partial^{2}}{\partial x^{2}} \frac{1}{r}\right]_{1} d V .
\end{aligned}
$$

Then, if $A^{\prime}$ is the coefficient in the Hölder condition on $\kappa$,

$$
\left|\Delta_{1}\right| \leqq 2 A^{\prime}\left\{\iiint_{\sigma} r_{1^{\lambda^{\prime}-3}} d V+\iiint_{\sigma} r_{2}^{\lambda^{\prime}-3} d V\right\} .
$$

From this follows the inequality

$$
\begin{aligned}
\left|\Delta_{1}\right| & \leqq 16 \pi A^{\prime} \int_{0}^{2 r_{12}} r_{1}^{\lambda^{\prime}-1} d r \\
& \leqq K_{1} r_{12}^{\lambda^{\prime}}
\end{aligned}
$$

for the first integral is not greater than the second.*

Passing to $\Delta_{2}$, we have

$$
\begin{aligned}
\Delta_{2}= & \left(\kappa_{1}-\kappa_{2}\right)\left[\frac{\partial^{2}}{\partial x^{2}} \iiint_{V} \frac{1}{r} d V\right]_{1}-\left(\kappa_{1}-\kappa_{2}\right)\left[\frac{\partial^{2}}{\partial x^{2}} \iiint_{\sigma} \frac{1}{r} d V\right]_{1} \\
& +\iiint_{V-\sigma}\left[\kappa-\kappa_{2}\right]\left[\left.\frac{\partial^{2}}{\partial x^{2}} \frac{1}{r}\right|_{2}-\left.\frac{\partial^{2}}{\partial x^{2}} \frac{1}{r}\right|_{1}\right] d V \\
= & \Delta_{21}-\Delta_{22}+\Delta_{23},
\end{aligned}
$$

where $\kappa_{1}$ and $\kappa_{2}$ are the values of $\kappa$ at $P_{1}$ and $P_{2}$. The coefficients of $\left(\kappa_{1}-\kappa_{2}\right)$ in $\Delta_{21}$ and $\Delta_{22}$ are bounded independently of $P_{1}$ and $r_{12}$, the first by Theorem XI and the second by the lemma just proved. Therefore,

$$
\begin{aligned}
& \left|\Delta_{21}\right| \leqq K_{2}{ }^{\lambda_{12}^{\gamma^{\prime}}}, \\
& \left|\Delta_{22}\right| \leqq K_{3}{ }^{\lambda_{12}^{\prime}}
\end{aligned}
$$

There remains $\Delta_{23}$ which may be written in the form

$$
\Delta_{23}=\int_{s_{1}}^{s_{2}} \iiint_{V-\sigma}\left[\kappa-\kappa_{2}\right] \frac{\partial^{3}}{\partial s \partial x^{2}} \frac{1}{r} d V d s,
$$

where the integration with respect to $s$ is from $P_{1}$ to $P_{2}$ along the segment joining them, and where the integrand is continuous in the field of integration. It follows that

\footnotetext{
* See Kellogg, Foundations of Potential Theory, loc. cit., p. 148, Lemma III.
} 


$$
\left|\Delta_{23}\right| \leqq 18 A^{\prime} \int_{s_{1}}^{s_{2}} \iiint_{V-\sigma} r_{2}^{\lambda^{\prime}} \frac{1}{r^{4}} d V d s,
$$

where $r$ is measured from a point of $P_{1} P_{2}$. Using the inequalities $r_{2}<2 r_{1}$, $r \geqq r_{1} / 2$, we have

$$
\begin{aligned}
\left|\Delta_{23}\right| & \leqq 576 A^{\prime} \int_{s_{1}}^{s_{2}} \iiint_{V-\sigma} r_{1}^{\lambda^{\prime}-4} d V d s \\
& \leqq 2304 \pi A^{\prime} \int_{s_{1}}^{s_{2}} \int_{2 r_{12}}^{\infty} r_{1}^{\lambda^{\prime}-2} d r d s \\
& \leqq \frac{2304 \pi A^{\prime}}{1-\lambda^{\prime}} r_{12}^{\lambda^{\prime}-1} \cdot r_{12} \leqq K_{4} r_{12}^{\lambda^{\prime}} .
\end{aligned}
$$

When the inequalities are assembled it appears that the second term satisfies a uniform Hölder condition in $V^{\prime}$ with exponent $\lambda^{\prime}$. Therefore, $\partial^{2} U / \partial x^{2}$ satisfies a uniform Hölder condition with exponent $\lambda^{\prime \prime}$. Similar reasoning holds for the other derivatives of second order, and the theorem holds for $n=2$ when $V^{\prime}$ is in $V$.

We turn now to the case in which $V^{\prime}$ is exterior to $V$ save for common boundary points on $\Sigma$. As we have seen, there is a neighborhood $N$ of any interior closed portion of $\Sigma$ with the property that through any point of $N$ there passes a normal to $\Sigma$ at an interior point of $\Sigma$. This neighborhood can be so chosen as to include all points of $\Sigma$ in $V^{\prime}$. We shall confine ourselves to the points common to $N$ and $V^{\prime}$, for since $U$ is harmonic at all other points of $V^{\prime}$, its derivatives satisfy Hölder conditions uniformly in this remaining portion of $V^{\prime}$.

Let $P_{1}$ be a point in $V^{\prime}$,--because of what has been said we shall from now on omit the specification that it be in $N$, - on the normal to $\Sigma$ at $p_{1}$. Let $P_{3}$ be a second point of $V^{\prime}$ such that the parallel through $P_{2}$ to $P_{1} p_{1}$ meets $\Sigma$ in the single point $p_{2}$, a situation always attainable by a uniform restriction on $\overline{P_{1} P_{2}}$. We refer $V$ to tangent-normal axes at $p_{1}$ and write

$$
\left.\frac{\partial^{2} U}{\partial x^{2}}\right|_{2}-\left.\frac{\partial^{2} U}{\partial x^{2}}\right|_{1}=\Delta_{1}+\Delta_{2}
$$

where

$$
\begin{aligned}
& \Delta_{1}=\kappa_{2}\left[\frac{\partial}{\partial x} \iint_{S} \cos (\nu, x) \frac{1}{r} d S\right]_{2}-\kappa_{1}\left[\frac{\partial}{\partial x} \iint_{S} \cos (\nu, x) \frac{1}{r} d S\right]_{1}, \\
& \Delta_{2}=\iiint_{V}\left(\kappa-\kappa_{2}\right)\left[\frac{\partial^{2}}{\partial x^{2}} \frac{1}{r}\right]_{2} d V-\iiint_{V}\left(\kappa-\kappa_{1}\right)\left[\frac{\partial^{2}}{\partial x^{2}} \frac{1}{r}\right]_{1} d V,
\end{aligned}
$$


$\kappa_{1}$ and $\kappa_{2}$ being the density at $p_{1}$ and $p_{2}$ respectively.

Since $\kappa$ is in $C^{\lambda^{\prime}}$, and since $\cos (\nu, x)$ is in $C^{\lambda}$ on $\Sigma$, we have, by Theorem VIII and by the reasoning of Lemma 1 ,

$$
\left|\Delta_{1}\right| \leqq K_{1}{ }^{\lambda \prime \prime}
$$

$K_{1}$ being an appropriate constant. Here $r_{12}$ is the distance $\overline{P_{1} P_{2}}$; and since the integrals are computed at $P_{1}$ and $P_{2}$ whereas $\kappa_{1}$ and $\kappa_{2}$ are the values of $\kappa$ at $p_{1}$ and $p_{2}$, it is important to notice. in the establishment of the above inequality that $\overline{p_{1} p_{2}}$ does not exceed the projection of $\overline{P_{1} P_{2}}$ times $k(=\max \sec \gamma)$, and hence

$$
\overline{p_{1} p_{2}} \leqq k \overline{P_{1} P_{2}} .
$$

In considering $\Delta_{2}$, we note that the points $P_{1}$ and $P_{2}$ may be thought of as not on $\Sigma$, for if a function, continuous in a closed region, satisfies a uniform Hölder condition in the interior, it satisfies the same uniform Hölder condition in the closed region.

Using now a sphere of radius $2 r_{12}$ about $p_{1}$ and calling the portion of $V$ in this sphere $\sigma$, we may write

$$
\Delta_{2}=\Delta_{21}+\Delta_{22}
$$

where in $\Delta_{21}$ the field of integration is $\sigma$, and in $\Delta_{22}, V-\sigma$. Then

$$
\begin{aligned}
\left|\Delta_{21}\right| \leqq 2 \iiint_{\sigma} \frac{\left|\kappa-\kappa_{2}\right|}{{\overline{P_{2}}}^{3}} d V \\
\quad+2 \iiint_{\sigma} \frac{\left|\kappa-\kappa_{1}\right|}{\bar{P}_{1}{ }^{3}} d V,
\end{aligned}
$$

$Q$ being the point of integration. If we let $r_{1}=p_{1} Q$, and $r_{2}=p_{2} Q$, we have

$$
\left|\Delta_{21}\right| \leqq 2 A^{\prime} k^{3}\left\{\iiint_{\sigma} r_{2}^{\lambda^{\prime}-3} d V+\iiint_{0} r_{1}^{\lambda^{\prime}-3} d V\right\},
$$

where $A^{\prime}$ is the coefficient in the Hölder condition on $\kappa$. From this follows the inequality

$$
\begin{aligned}
\left|\Delta_{21}\right| & \leqq 16 \pi A^{\prime} k^{3} \int_{0}^{2 r_{12}} r_{1^{\lambda^{\prime}-1}} d r \\
& \leqq K_{2} r_{12},
\end{aligned}
$$

since the first integral is not greater than the second.

Passing to $\Delta_{22}$, we have 


$$
\begin{aligned}
\Delta_{22}= & \iiint_{V-\sigma}\left(\kappa-\kappa_{2}\right)\left[\frac{\partial^{2}}{\partial x^{2}} \frac{1}{r}\right]_{2} d V-\iiint_{V-\sigma}\left(\kappa-\kappa_{1}\right)\left[\frac{\partial^{2}}{\partial x^{2}} \frac{1}{r}\right]_{1} d V \\
= & \left(\kappa_{1}-\kappa_{2}\right) \iiint_{V-\sigma}\left[\frac{\partial^{2}}{\partial x^{2}} \frac{1}{r}\right]_{1} d V \\
& +\iiint_{V-\sigma}\left(\kappa-\kappa_{2}\right)\left[\left.\frac{\partial^{2}}{\partial x^{2}} \frac{1}{r}\right|_{2}-\left.\frac{\partial^{2}}{\partial x^{2}} \frac{1}{r}\right|_{1}\right] d V \\
= & \Delta_{221}+\Delta_{222} .
\end{aligned}
$$

We may write

$$
\Delta_{221}=\left(\kappa_{1}-\kappa_{2}\right) \frac{\partial}{\partial x} \iint_{S^{\prime}} \cos (\nu, x) \frac{1}{r} d S,
$$

where $S^{\prime}$ is the boundary of $V-\sigma$, and $r$ is measured from $P_{1}$. Since $\kappa$ satisfies a uniform Hölder condition with exponent $\lambda^{\prime}, \Delta_{221}$ will satisfy an inequality of the desired form if the derivative of the simple spread is bounded. That this is so may be seen by writing it in the form

$$
\begin{aligned}
\frac{\partial}{\partial x} \iint_{S^{\prime}} \cos (\nu, x) \frac{1}{r} d S & =\frac{\partial}{\partial x} \iint_{S} \cos (\nu, x) \frac{1}{r} d S-\frac{\partial}{\partial x} \iint_{\Sigma_{1}} \cos (\nu, x) \frac{1}{r} d S \\
& +\frac{\partial}{\partial x} \iint_{\Sigma_{2}} \cos (\nu, x) \frac{1}{r} d S
\end{aligned}
$$

where $\Sigma_{1}$ is the portion of $\Sigma$ contained in $\sigma$, and $\Sigma_{2}$ is the rest of the boundary of $\sigma$.

The first term on the right, being a derivative in a fixed direction of the potential of a simple spread with density of class $C^{\lambda}$, is continuous, and therefore bounded in $V^{\prime}$. The same reasoning cannot be applied to the remaining two terms because of the presence of edges at distances from $p_{1}$ which approach 0 with $r_{12}$. But they are, nevertheless, bounded, uniformly as to $P_{1}$ and $p_{1}$. This may be seen as follows.

In the second term $|\cos (\nu, x)| \leqq A r_{1}{ }^{\lambda}$, where $r_{1}$ is the distance from $p_{1}$ to the integration point, because $\Sigma$, and hence $\Sigma_{1}$, are of class $C^{\lambda}$, and since $\cos (\nu, x)$ vanishes at $p_{1}$ on account of the position of the axes. Hence

$$
\begin{aligned}
\left|\frac{\partial}{\partial x} \iint_{\Sigma_{1}} \cos (\nu, x) \frac{1}{r} d S\right| & \leqq \iint_{\Sigma_{1^{\prime}}}|\cos (\nu, x)| \frac{1}{r^{2}} \sec \gamma d S^{\prime} \\
& \leqq k^{1+\lambda} A \iint_{\Sigma} r^{\prime \lambda-2} d S^{\prime}
\end{aligned}
$$


where $r^{\prime}$ is the projection of $r$ and $r_{1}$ on the $(x, y)$-plane, where $k=\max \sec \gamma$ on $\Sigma_{1}$ and the integration is over the projection of $\Sigma_{1}$. The last integral is uniformly convergent and vanishes with $r_{12}$.

In the third term, we have $2 r_{12} \leqq k r$, where $2 r_{12}$ is the radius of $\Sigma_{2}$ and $k=\max \sec \gamma$. Hence, the term is not greater in absolute value than

$$
\iint_{\Sigma_{2}} \frac{1}{r^{2}} d S \leqq k^{2} \iint_{\Sigma_{2}} \frac{1}{\left(2 r_{12}\right)^{2}} d S \leqq 4 \pi k^{2} .
$$

Thus

$$
\left|\Delta_{221}\right| \leqq K_{3} r_{12}^{\lambda^{\prime}}
$$

Finally, we have to consider $\Delta_{222}$, which may be written in the form

$$
\Delta_{222}=\int_{s_{1}}^{s_{2}} \iiint_{V-\sigma}\left(\kappa-\kappa_{2}\right) \frac{\partial^{3}}{\partial s \partial x^{2}} \frac{1}{r} d V d s,
$$

where the integration with respect to $s$ is from $P_{1}$ to $P_{2}$ along the segment joining them, and where the integrand is continuous in the field of integration.

Let $a$ denote a constant such that in the sphere of radius $a$ about $p_{1}$ the angle between any two normals never exceeds $\pi / 6 ; a$ can be selected independently of $p_{1}$, because of the uniform continuity of the direction cosines of the normal to $\Sigma$. If we restrict $r_{12}$ to be less than $a / 4$, we may confine ourselves to the portion $v$ of $V$ between the sphere of radius $a$ about $p_{1}$, and the sphere $\sigma$, of radius $2 r_{12}$; for, in the remaining field, the integrand in $\Delta_{222}$ is uniformly bounded, and the corresponding integral, accordingly, does not exceed in absolute value a constant times $r_{12}$. For the rest,

$$
\begin{aligned}
\left|\int_{s_{1}}^{s_{2}} \iiint_{v}\left(\kappa-\kappa_{2}\right) \frac{\partial^{3}}{\partial s \partial x^{2}} \frac{1}{r} d V d s\right| & \leqq 18 A^{\prime} \int_{s_{1}}^{s_{2}} \iiint_{v} r_{2^{\lambda^{\prime}}} \frac{1}{r^{4}} d V d s \\
& \leqq 18 A^{\prime} \int_{s_{1}}^{s_{2}} \iiint_{v} r_{2^{\lambda^{\prime}-4}}\left(\frac{r_{2}}{r}\right)^{4} d V d s,
\end{aligned}
$$

where $r$ is measured from a point of the segment $P_{1} P_{2}$, and $r_{2}$ is measured from $p_{2}$. For any fixed position of the point of integration, $r_{2} / r$ is greatest when $r$ is measured as nearly as possible perpendicular to the normal to $\Sigma$ at $p_{1}$, in which case

$$
r_{2} \leqq\left(r+P_{1} P_{2}\right) \max \sec \gamma \leqq \frac{2}{3^{1 / 2}}\left(r+r_{12}\right)
$$

Moreover, 


$$
r \geqq \frac{2 r_{12}}{\max \sec \gamma}-r_{12} \geqq\left(3^{1 / 2}-1\right) r_{12} .
$$

Hence,

$$
\frac{r_{2}}{r} \leqq \frac{2}{3^{1 / 2}}\left(1+\frac{r_{12}}{r}\right)<3 .
$$

Accordingly,

$$
\begin{aligned}
\left|\int_{s_{1}}^{s_{2}} \iiint_{v}\left(\kappa-\kappa_{2}\right) \frac{\partial^{3}}{\partial s \partial x^{2}} \frac{1}{r} d V d s\right| & \leqq 1458 A^{\prime} \int_{s_{1}}^{s_{2}} \iiint_{v} r_{2}^{\lambda^{\prime}-4} d V d s \\
& \leqq 5832 \pi A^{\prime} \int_{s_{1}}^{s_{2}} \int_{2 r_{1}}^{2 a} r_{2}^{\lambda^{\prime}-2} d r_{2} d s \\
& \leqq \frac{5832 \pi A^{\prime}}{1-\lambda^{\prime}} r_{12}^{\lambda^{\prime}-1} \cdot r_{12} \\
& \leqq K_{4} r_{12}^{\lambda^{\prime}} .
\end{aligned}
$$

When the inequalities are assembled, it appears that $\partial^{2} U / \partial x^{2}$ satisfies a uniform Hölder condition in $V^{\prime}$ with exponent $\lambda^{\prime \prime}$. The reasoning requires no modification in the case of other derivatives of the second order, provided one direction is tangential. In the case of $\partial^{2} U / \partial z^{2}$, with the above orientation of the axes, the only point at which modification is necessary is in the proof that the second term in the expression (12) is bounded. For, cos $(\nu, z)=1$ at $p_{1}$. In this case, however, the term becomes, when we use the projection $\Sigma_{1}^{\prime}$ of $\Sigma_{1}$ on the $(x, y)$-plane, as the field of integration,

$$
\frac{\partial}{\partial z} \iint_{\Sigma_{1^{\prime}}} \frac{1}{r} d S^{\prime}=\iint_{\Sigma_{1^{\prime}}} \frac{\zeta-z}{r^{3}} d S^{\prime} .
$$

This can be shown to be bounded, independently of $P_{1}, p_{1}$ and $r_{12}$, by comparing it with the same derivative of a spread of unit density on the flat region $\Sigma_{1}^{\prime}$,

$$
\iint_{\Sigma_{1^{\prime}}}-\frac{z}{\rho^{3}} d S^{\prime}, \rho^{2}=\xi^{2}+\eta^{2}+z^{2},
$$

and noting that $\rho / r$ lies uniformly between two positive bounds.

When the details are supplied, the proof of Theorem XII for $n=2$ is complete.

For $n>2$, we use the identity (11) and assume that the theorem has been established for derivatives of order lower than $n$. Then the first term in (11) 
has continuous derivatives of order $n-1$ in $V^{\prime}$ which satisfy there a uniform Hölder condition with exponent $\lambda^{\prime \prime}$, by Theorem VIII. The same is true of the second term, by our assumption about the derivatives of order lower than $n$. Therefore, the theorem is true for derivatives of order $n$. Since it has been established for $n=2$, it follows that it holds for any $n \geqq 2$.

12. Logarithmic potentials. The potential of a logarithmic distribution on a plane curve can be interpreted as the potential of a distribution on an infinite cylinder with elements perpendicular to the plane of the curve. Furthermore, the potential of the distribution on the portion $S$ of the cylinder, outside two planes parallel to and on either side of the plane of the curve, is continuous and has continuous derivatives of all orders at all points of the plane of the curve.* The situation is the same for logarithmic double distributions and for logarithmic distributions on plane areas.

Knowing this, we can see immediately that all the theorems established in this paper for surface or volume distributions hold also for logarithmic distributions on plane curves or areas, without alteration other than the appropriate changes in dimensionality.

* Kellogg, Foundations of Potential Theory, loc. cit., p. 174.

Radcliffe College, Cambridge, Mass. 